\title{
A Measurement Based Shadow Fading Model for Vehicle-to-Vehicle Network Simulations
}

\author{
Taimoor Abbas, ${ }^{1}$ Katrin Sjöberg, ${ }^{2,3}$ Johan Karedal, ${ }^{1}$ and Fredrik Tufvesson ${ }^{1}$ \\ ${ }^{1}$ Department of Electrical and Information Technology, Lund University, P.O. Box 118, 22100 Lund, Sweden \\ ${ }^{2}$ The Centre for Research on Embedded Systems, Halmstad University, Halmstad, Sweden \\ ${ }^{3}$ Department of Advanced Technology and Research, Volvo Group Trucks Technology (GTT), Götaverksg. 10, \\ 40508 Gothenburg, Sweden
}

Correspondence should be addressed to Taimoor Abbas; taimoor.abbas@gmail.com

Received 16 February 2015; Accepted 17 May 2015

Academic Editor: Christoph F. Mecklenbräuker

Copyright (C) 2015 Taimoor Abbas et al. This is an open access article distributed under the Creative Commons Attribution License, which permits unrestricted use, distribution, and reproduction in any medium, provided the original work is properly cited.

\begin{abstract}
The vehicle-to-vehicle (V2V) propagation channel has significant implications on the design and performance of novel communication protocols for vehicular ad hoc networks (VANETs). Extensive research efforts have been made to develop V2V channel models to be implemented in advanced VANET system simulators for performance evaluation. The impact of shadowing caused by other vehicles has, however, largely been neglected in most of the models, as well as in the system simulations. In this paper we present a shadow fading model targeting system simulations based on real measurements performed in urban and highway scenarios. The measurement data is separated into three categories, line-of-sight (LOS), obstructed line-of-sight (OLOS) by vehicles, and non-line-of-sight due to buildings, with the help of video information recorded during the measurements. It is observed that vehicles obstructing the LOS induce an additional average attenuation of about $10 \mathrm{~dB}$ in the received signal power. An approach to incorporate the LOS/OLOS model into existing VANET simulators is also provided. Finally, system level VANET simulation results are presented, showing the difference between the LOS/OLOS model and a channel model based on Nakagami- $m$ fading.
\end{abstract}

\section{Introduction}

Vehicle-to-Vehicle (V2V) communication allows vehicles to communicate directly with minimal latency. The primary objective with the message exchange is to improve active onroad safety and situation awareness, for example, collision avoidance, traffic rerouting, and navigation. The propagation channel in V2V networks is significantly different from that in cellular networks because V2V employs an ad hoc network topology, both transmitter (TX) and receiver (RX) are highly mobile, and TX/RX antennas are situated on approximately the same height and close to the ground level. Thus, to develop an efficient and reliable system a deep understanding of V2V channel characteristics is required [1].

A number of $\mathrm{V} 2 \mathrm{~V}$ measurements have been performed to study the statistical properties of $\mathrm{V} 2 \mathrm{~V}$ propagation channels [2-6]. Signal propagation over the wireless channel is often divided by three statistically independent phenomena named deterministic path loss, small-scale fading, and large-scale or shadow fading [7]. Path loss is the expected (mean) loss at a certain distance compared to the received power at a reference distance. The signal from the TX can reach the RX via several propagation paths or the multipath components (MPC), which have different amplitudes and phases. The change in the signal amplitude due to constructive or destructive interference of the different MPCs is classified as small-scale fading. Finally, obstacles in the propagation paths of one or more MPCs can cause large attenuation and the effect is called shadowing. Shadowing gives rise to largescale fading and it occurs not only for the line-of-sight (LOS) component but also for any other major MPC. Understanding all of these phenomena is equally important to characterize the V2V propagation channel.

In real scenarios there can be light to heavy road traffic, involving vehicles with variable speeds and heights, and there are sometimes buildings around the roadside. Hence, it might be the case that the LOS is partly or completely blocked by another vehicle or a house. The received power depends very 
much on the propagation environment and the availability of LOS. Moreover, in [8] it is reported that, in the absence of LOS, most of the power is received by single bounce reflections from physical objects. Therefore, for a realistic simulation and performance evaluation it is important that the channel parameters are separately characterized for LOS and non-LOS conditions.

A number of different V2V measurement based studies with their extracted channel parameters are summarized in [9]. For most of the investigations mentioned in [9], it is assumed that the LOS is available for the majority of the recorded snapshots. Thus, the samples from both the LOS and non-LOS cases are lumped together for modeling, which is somewhat unrealistic, especially for larger distances. The LOS path being blocked by buildings greatly impacts the reception quality in situations when vehicles are approaching the street intersection or road crossings. The buildings at the corners influence the received signal not only by blocking the LOS but also they act as scattering points which helps to capture more power in the absence of LOS [10]. A few measurement results for a non-LOS (NLOS) environment are available [11-19] in which the path loss model is presented for different types of street crossings.

In addition to the NLOS situation, the impact of neighboring vehicles can not be ignored. In [5], it is reported that the received signal strength degrades on the same patch of an open road in heavy traffic hours as compared to when there is light traffic. These observed differences can only be related to other vehicles obstructing LOS since the system parameters remained the same during the measurement campaign. Similarly, Zang et al. in [20] presented an abstract error model in which the LOS and NLOS cases are separated using a thresholding distance. It is stated that the signals will experience more serious fading in crowded traffic scenario when the distance between the TX and RX is larger than the thresholding distance.

In [21, 22], it is shown that the vehicles (as obstacles) have a significant impact on LOS obstruction in both dense and sparse vehicular networks, implying that shadowing caused by other vehicles cannot be ignored in V2V channel models. To date, in majority of the findings for $\mathrm{V} 2 \mathrm{~V}$ communications except [21, 22], the shadowing impact of vehicles has largely been neglected when modeling the path loss. It is important to model vehicles as obstacles; ignoring this can lead to unrealistic assumptions about the performance of the physical layer, in terms of both received signal power and interference levels, which in turn can affect the behavior of higher layers of $\mathrm{V} 2 \mathrm{~V}$ systems.

In order to characterize the channel parameters separately for LOS and non-LOS conditions V2V communication links in this paper are categorized into the following three groups:

(i) Line-of-sight (LOS) is the situation when there is an optical line-of-sight between the TX and the RX.

(ii) Obstructed LOS (OLOS) is the situation when the LOS between the TX and RX is obstructed completely or partially by another vehicle. (iii) Non-LOS (NLOS) is the situation when a building between the TX and RX completely block the LOS as well as many other significant MPCs.

The channel properties for LOS, OLOS, and NLOS are distinct, and their individual analysis is required. No path loss model, except a geometry based channel model published recently [23], is today available dealing with all three cases in a comprehensive way.

The main contribution of this paper is a shadow fading channel model (LOS/OLOS model) based on real measurements in highway and urban scenarios distinguishing between LOS and OLOS. The model targets vehicular ad hoc network (VANET) system simulations. We also provide a solution on how to incorporate the LOS/OLOS model in a VANET simulator. We model the temporal correlation of shadow fading as an autoregressive process. Finally, simulation results are presented where the results obtained from the LOS/OLOS model are compared against Cheng's model [24], which is also based on an outdoor channel sounding measurement campaign performed at $5.9 \mathrm{GHz}$. The reason of choosing Cheng's model for comparison is that the Cheng's model do not classify measured data as LOS, OLOS, and NLOS, but it represents both the small-scale fading and the shadowing by the Nakagami- $m$ model.

The remainder of the paper is organized as follows. Section 2 outlines the outdoor V2V measurements and explains the methods for separating LOS, OLOS, and NLOS data samples which serves as first step to model the effects of shadow fading. It also includes the derivation of path loss and modeling of shadow fading in LOS and OLOS cases as log-normal distribution. The channel model is provided in Section 3. First, the extension in traffic mobility models is suggested to include the effect of large-scale fading, and then the path loss model is presented and parameterized based on the measurements. VANET simulation results are discussed in Section 4. Finally, Section 5 concludes the paper.

\section{Methodology}

2.1. Measurement Setup. Channel measurement data was collected using the RUSK-LUND channel sounder, which performs multiple-input multiple-output (MIMO) measurements based on the switched array principle. The measurement bandwidth was $200 \mathrm{MHz}$ centered around a carrier frequency of $5.6 \mathrm{GHz}$ and total $N_{f}=641$ frequency points. For the analysis the complex time-varying channel transfer function $H(f, t)$ was measured for two different time durations: short term (ST), $25 \mathrm{~s}$, and long term (LT), $460 \mathrm{~s}$. The short-term and long-term channel transfer functions were composed of total $N_{t}=49152$ and $N_{t}=4915$ time samples, sampled with a time spacing of $\Delta t=0.51 \mathrm{~ms}$ and $\Delta t=94.6 \mathrm{~ms}$, respectively. The test signal length was set to $3.2 \mu \mathrm{s}$.

Two standard $1.47 \mathrm{~m}$ high station wagons, Volvo V70 cars, were used during the measurement campaign. An omnidirectional antenna was placed on the roof of the TX and RX vehicles, taped on a styrofoam block that in turn was taped sideways to the shark fin on the center of the roof, and 


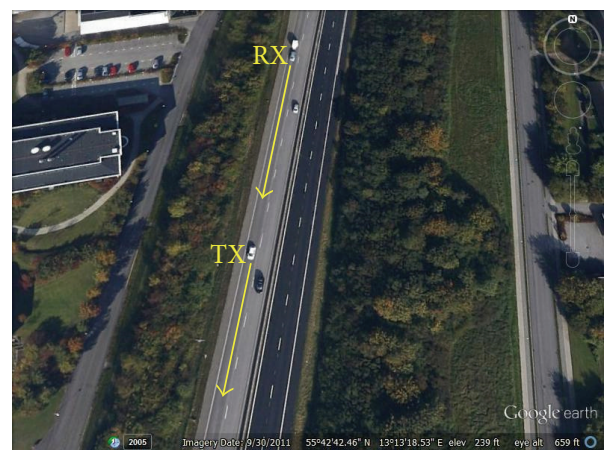

FIgURE 1: Aerial shot of the measurement route on a highway between the cities of Lund and Malmö, Sweden ( $55^{\circ} 43^{\prime} 46^{\prime \prime}, \mathrm{E}$ $\left.13^{\circ} 14^{\prime} 33^{\prime \prime}\right)$.

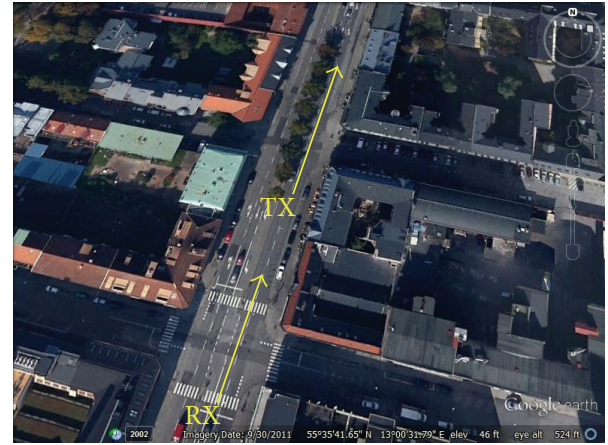

FIGURE 2: Aerial shot of the measurement route on an urban road in Malmö, Sweden (N $55^{\circ} 35^{\prime} 41^{\prime \prime}$, E $\left.13^{\circ} 0^{\prime} 31^{\prime \prime}\right)$.

$360 \mathrm{~mm}$ from the back edge of the roof. Videos were taken through the windscreen of each TX/RX car and GPS data was also logged during each measurement. Video recordings and GPS data together with the measurement data were used in the postprocessing to identify the LOS, OLOS, and NLOS conditions and important scatterers and to keep track of the distance between the two cars. The videos were synchronized to the measurements (for details see [25]).

2.2. Measurement Routes. Eight routes in two different propagation environments were chosen with differences in their traffic densities, roadside environments, number of scatterers, pedestrians, and houses along the road side. All measurements were conducted in and between the cities Lund and Malmö, in southern Sweden. Aerial shots of the measurement routes are shown in Figures 1 and 2.

2.2.1. Highway. Measurements were performed when both the TX and RX cars were moving in a convoy at a speed of $22-25 \mathrm{~m} / \mathrm{s}(80-90 \mathrm{~km} / \mathrm{h})$, on a 2-lane (in each direction) highway, between the cities of Lund and Malmö, Sweden. The traffic density was varying on both sides of the road from low to high traffic. Along the roadside there were trees, vegetation, road signs, street lights, and few buildings situated at random distances. The direction of travel was separated by a $(\approx 0.5 \mathrm{~m}$
TABLE 1: Distance traveled in LOS, OLOS, and NLOS conditions.

\begin{tabular}{lcccccc}
\hline & Scenario & Total & Min & Max & Mean & Median \\
\hline \multirow{2}{*}{ LOS (m) } & Highway & 6622 & 24.4 & 2157 & 299 & 125 \\
& Urban & 5477 & 0.95 & 519 & 84.6 & 35.3 \\
\hline \multirow{2}{*}{ OLOS (m) } & Highway & 10752 & 18.6 & 2298 & 467 & 150 \\
& Urban & 2429 & 2.4 & 656 & 39.8 & 20.5 \\
\hline \multirow{2}{*}{ NLOS (m) } & Urban & 415 & - & - & - & - \\
\hline
\end{tabular}

tall) concrete wall whereas the outer boundary of the road was guarded by a metallic rail.

2.2.2. Urban. Measurements were performed when both the $\mathrm{TX}$ and RX cars were moving in a convoy as well as in the opposite directions, in densely populated areas in Lund and Malmö. The TX and RX cars were moving with different speeds, between 0 and $14 \mathrm{~m} / \mathrm{s}(0-50 \mathrm{~km} / \mathrm{h})$, depending on the traffic situation. The streets were either single or double lane (12-20 m wide) lined with 2-4 storied buildings. There were trees on either side and sidewalks on both sides of the streets. Moreover, there were road signs, street lights, bicycles, and many parked cars, usually on both sides of the street. The streets were occupied with a number of moving vehicles as well as few pedestrians walking on the sidewalks.

In total $3 \mathrm{ST}$ and $2 \mathrm{LT}$ measurements for highway and $7 \mathrm{ST}$ and 4LT measurements for urban-convoy were performed. During each measurement, the LOS was often obstructed by other cars, taller vans, trucks, buses, or houses at the street corners.

2.3. LOS, OLOS, and NLOS Separation. The measured channel transfer functions were first separated as LOS, OLOS, and NLOS for all the measurements. The geometric information available from the video recording from the measurements has served as the foundation for the separation process. To distinguish the LOS samples from OLOS and NLOS samples, we define LOS condition as when it is possible for one of the cameras to see the middle of the roof of the other vehicle. Otherwise, the LOS is categorized as blocked. The blocked LOS situation is further categorized into two groups as defined previously: OLOS and NLOS. The recorded videos contain 25 frames per second and are synchronized to the measurements. Therefore, a frame-by-frame evaluation of video data is performed to separate the data for LOS, OLOS, and NLOS cases.

During the whole measurement run, the TX-RX link transited between LOS, OLOS, and NLOS states a number of times, that is, LOS-to-OLOS, 61 times in urban and 23 times in highway scenario, and, similarly, LOS-to-NLOS, 4 times in urban and 0 times in highway scenario. Each time the TXRX pair is in either LOS, OLOS, or NLOS state, it remains in that state for some time interval and travel a certain distance relative to the speed vehicles. In Table 1 the traveled distances for both scenarios, urban and highway, are tabulated together with the distances where TX/RX were in LOS, OLOS, and NLOS, respectively. The Cumulative Distribution Function (CDF) of these LOS/OLOS distance intervals is shown in 


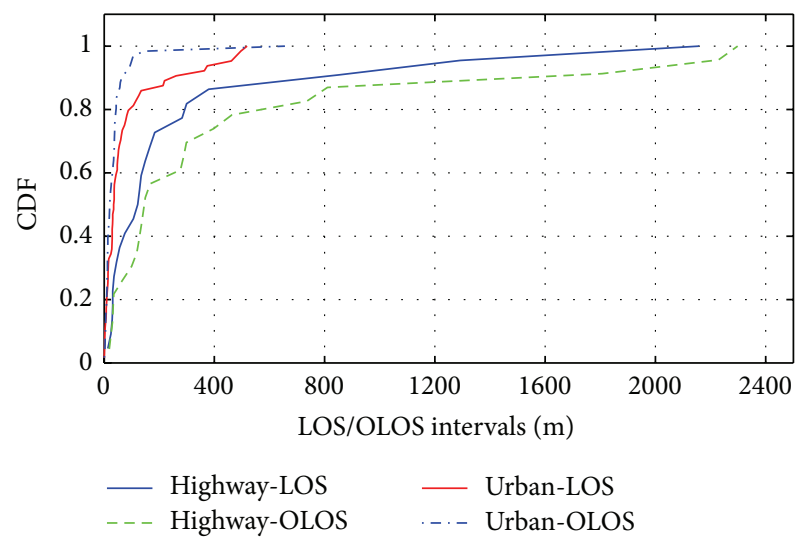

FIgUre 3: Cumulative Distribution Function (CDF) of LOS and OLOS distance intervals for all measurements: (a) highway scenario and (b) urban scenarios.

Figure 3. No transition took place from OLOS-to-NLOS. The NLOS does not usually occur on highways, and the data samples for NLOS data in urban measurements are too few to be plotted as a CDF.

2.4. Path loss Derivation. The time varying power-delayprofile (PDP) is derived for each time sample in order to determine the path loss. The effect of small scale fading is eliminated by averaging the time varying PDP over $N_{\text {avg }}$ number of time samples; the averaged-PDP (APDP) is given by [26] as

$$
P_{h}\left(t_{k}, \tau\right)=\frac{1}{N_{\text {avg }}} \sum_{n=0}^{N_{\text {avg }}-1}\left|h\left(t_{k}+n \Delta t, \tau\right)\right|^{2},
$$

for $t_{k}=0, N_{\mathrm{avg}} \Delta t, \ldots,\left\lfloor N_{t} / N_{\mathrm{avg}}-1\right\rfloor N_{\mathrm{avg}} \Delta t$, where $h\left(t_{k}+\right.$ $n \Delta t, \tau)$ is the complex time varying channel impulse response derived by an inverse Fourier transform of a channel transfer function $H(f, t)$ for a single-input single-output (SISO) antenna configuration. The $N_{\text {avg }}$ corresponds to the movement of the TX and RX by $s=15 \lambda$ and is calculated by $N_{\text {avg }}=s / v \Delta t$, where $\Delta t$ is the time spacing between snapshots and $v$ is the velocity of TX and RX given in each scenario description. $N_{\text {avg }} \Delta t$ equals $32 \mathrm{~ms}$ and $71 \mathrm{~ms}$ for highway and urban scenarios, respectively, and they are chosen such that the wide-sense stationary (WSS) assumption is valid over $N_{\text {avg }}$ snapshots [27].

The noise thresholding of each APDP is performed by suppressing all signals with power below the noise floor, that is, noise power plus a $3 \mathrm{~dB}$ additional margin, to zero. The noise power is determined from the part of PDP, at larger delays, where no contribution from the transmitted signal is present. Thus, the zeroth-order moment of the noise thresholded, small-scale averaged APDPs gives the averaged channel gain for each link as

$$
G_{h}\left(t_{k}\right)=\sum_{\tau} P_{h}\left(t_{k}, \tau\right)
$$

where $\tau$ is the propagation delay.
Finally, the antenna influence and other implementation losses such as cable attenuation and the effect of the low-noise amplifier (LNA) were removed from the measured gains. The azimuth antenna pattern was almost omnidirectional with variations of about $2 \mathrm{~dB}$ and peak gain of about $3.7 \mathrm{dBi}$, which was measured in an anechoic chamber. The distance dependent path loss $\operatorname{PL}(d)$ is then calculated using the following equation:

$$
\mathrm{PL}(d)=2 G_{a}-P_{\mathrm{IL}}-10 \log _{10} G_{h}(d),
$$

where $G_{h}(d)$ is the distance dependent channel gain, which is obtained by matching the time dependent channel gain $G_{h}\left(t_{k}\right)$ to its corresponding distance $d$ between TX and RX at time instant $t_{k}, G_{a}$ is the antenna gain, and $P_{\mathrm{IL}}$ is the implementation loss.

GPS data, recorded during the measurements, was used to find the distance between TX and RX, which corresponds to the propagation distance of first arriving path for each time sample in the presence of LOS. The time resolution of the GPS data was limited to one GPS position/second. Thus, to make GPS data sampling rate equal to the time snapshots, interpolation of the GPS data was performed with a cubic spline method. The distance obtained from the GPS data was further validated, later, by tracking the first arrived $\mathrm{MPC}$, in the presence of LOS, with a high resolution tracking algorithm [28].

2.5. Large-Scale or Shadow Fading. As explained earlier the effect of small scale fading is removed by averaging the received signal power over a distance of $15 \lambda$. The averaged envelope is a random variable due to the large-scale variations caused by the shadowing from large objects such as building and vehicles. The most widely accepted approach is to model the large-scale variations with a log-normal distribution function [7, 29].

For the analysis of large-scale variations the distance dependent channel gain $G_{h}(d)$ is divided into log-spaced distance bins and the distribution of the data associated to each bin is studied independently with and without the separation of LOS, OLOS, and NLOS data. Before the data separation it was observed that a log-normal distribution does not provide a good approximation of the data, as often anticipated, as shown in Figures 4(a) and 4(d). Moreover, an additional attenuation was observed, which made the spread in the channel gain large and the spread was different for different distance bins. The conclusion was drawn that the attenuation could possibly be associated to the LOS obstruction. Therefore, it was important to separate the data for the LOS, OLOS, and NLOS situations. The analysis of the separated data sets showed that the large-scale variations for both LOS and OLOS can be modeled as log-normal distribution (see, e.g., Figures 4(b) and 4(f)) with an offset of almost $10 \mathrm{~dB}$ in their mean. In highway and open scenarios even higher losses are expected due to obstructed LOS especially when the two cars have communication distance less than $80 \mathrm{~m}$. This observation is in line with the independent observations presented in [22]. In which it is found that a 


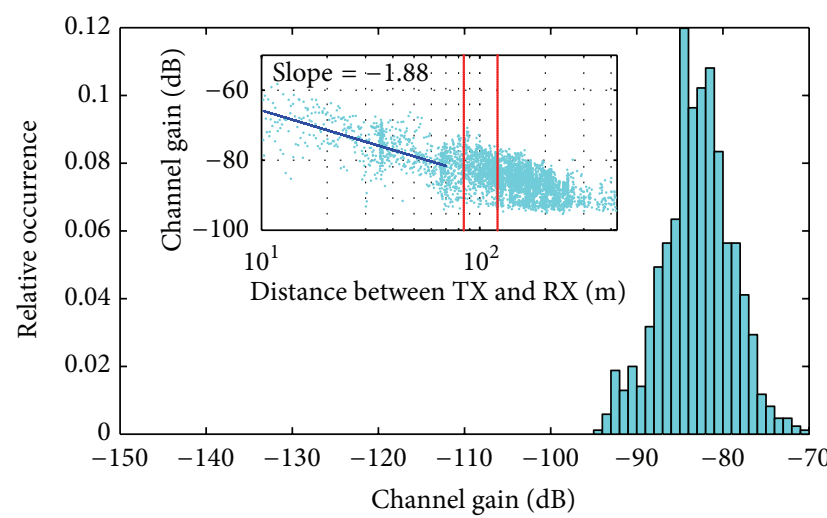

(a)

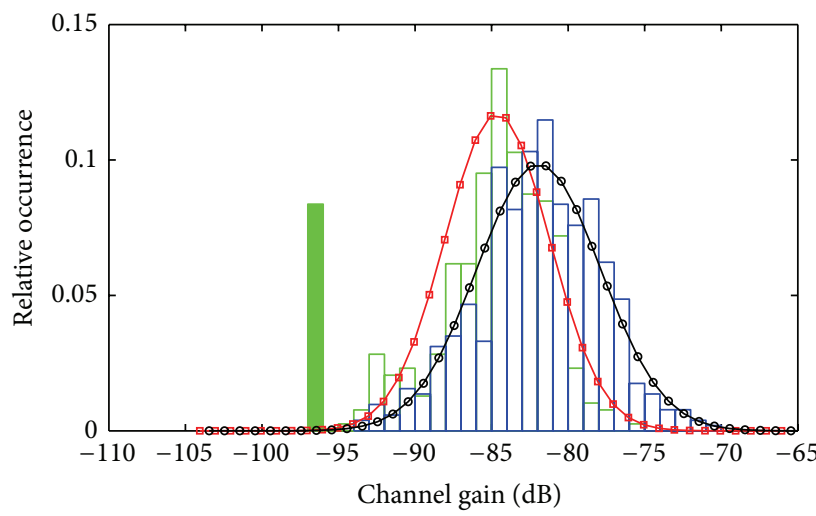

(b)

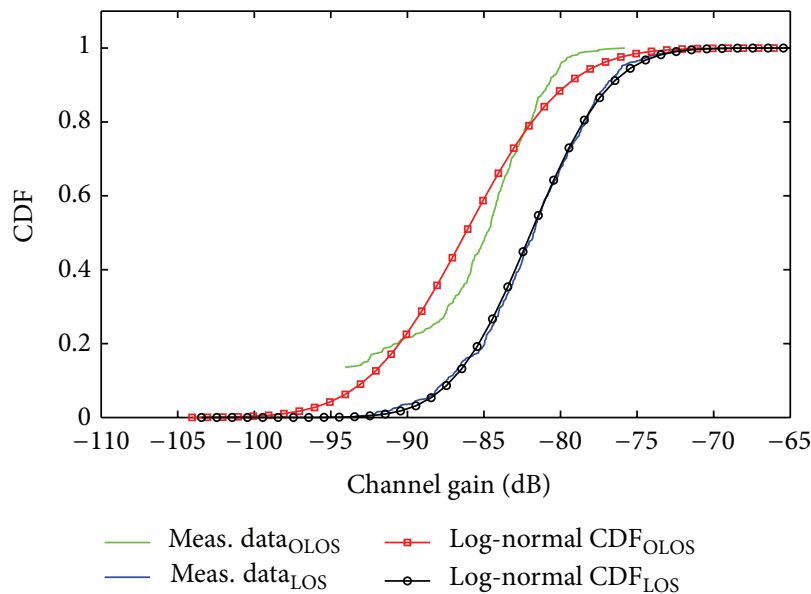

(c)

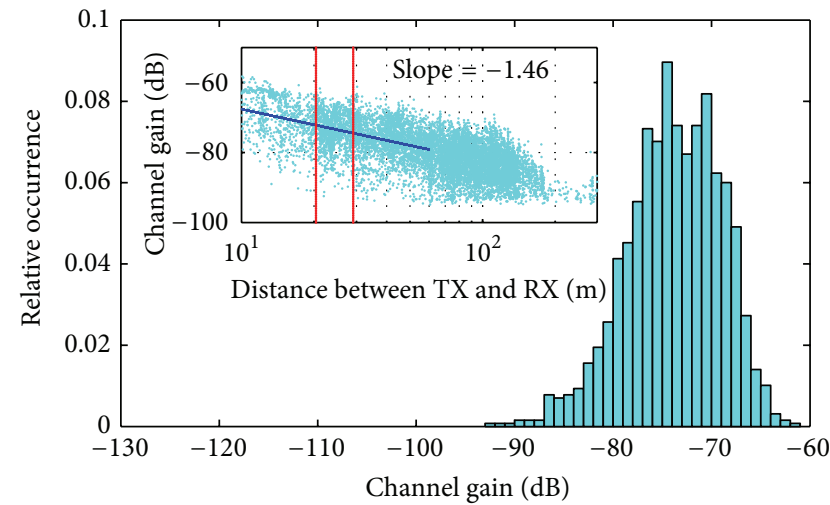

(d)

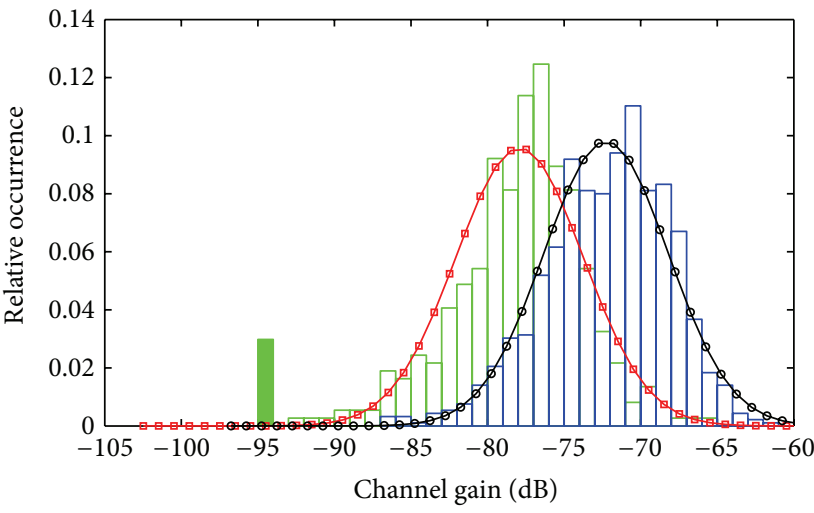

Below noise threshold $\rightarrow$ Normal PDF OLOS Meas, data OLOS $\rightarrow$ Normal PDF LOS

Meas. data LOS

(e)

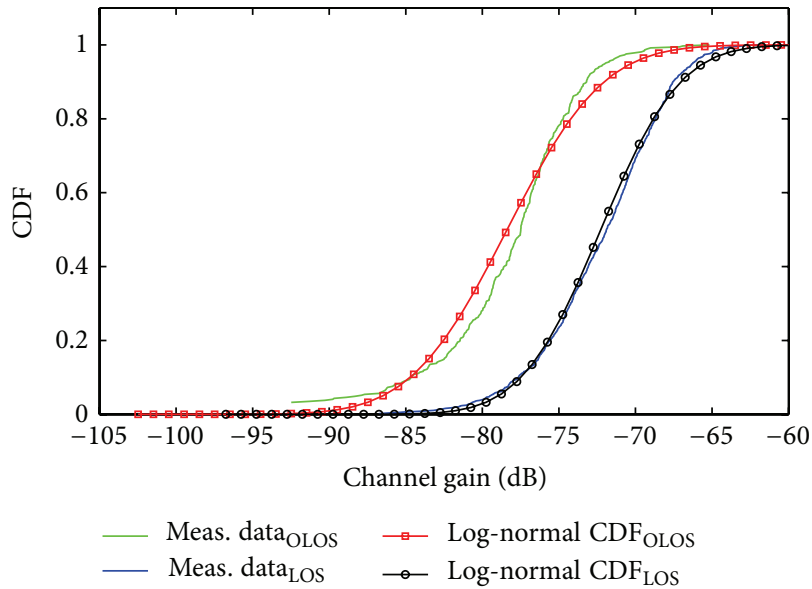

(f)

FIGURE 4: a, b, and c represent highway data, and d, e, and f represent urban data. In (a) and (d) inset plots show channel gain for the overall measurement data as a function of direct distance between TX and RX. The slopes are provided up to the distance where no sample is below the noise. Large plot is the histogram of channel gains taken from log spaced distance bin, 20.4-29.1 $\mathrm{m}$ in (a) and 84.6-121 $\mathrm{m}$ in (d), marked by vertical lines in the inset plot. The LOS and OLOS data is treated together in these figure; (b) and (e) show histogram of the same channel gains shown in (a) and (d), when separated as LOS and NLOS, pdf fitting the Gaussian distribution; (c) and (f) show CDF fitting the log-normal distribution with $95 \%$ confidence interval. 
single obstruction at the communication distance of about $10 \mathrm{~m}$ can cause an additional attenuation of about $20 \mathrm{~dB}$.

The channel gain in the OLOS condition momentarily falls below the noise floor of the channel sounder and power levels of samples below the noise threshold cannot be detected correctly. However, the OLOS data in each bin for shorter distances, with no missing samples, fits well to a lognormal distribution, and the assumption that the data continues to follow a log-normal distribution for larger distance bins where the observed data is incomplete is made. Moreover, the exact count of missing samples is also available, which can be used to estimate the overall data distribution. To get the mean and variance of Gaussian distributed LOS and OLOS data, the maximum likelihood estimates (MLE) of scale and position parameters from incomplete data were computed by using the method in [30], in which Dempster et al. presented a broadly applicable algorithm that iteratively computes MLE from incomplete data via expectation maximization (EM).

\section{Channel Model}

In this section a shadow fading model (LOS/OLOS model) for VANET simulations is provided. This model targets network simulation, where there is a need for a realistic model taking shadowing effects into account but still with a reasonable complexity.
3.1. Path loss Model. The measurement data is split into three data sets: LOS, OLOS, and NLOS. The parameters of the path loss model are extracted only for the LOS and the OLOS data sets, whereas not enough data is available to model the path loss for the third data set, NLOS.

The measured channel gain for LOS and OLOS data for the highway and the urban scenario is shown as a function of distance in Figures 5 and 6, respectively. A simple logdistance power law [7] is often used to model the path loss to predict the reliable communication range between the transmitter and the receiver. The generic form of this logdistance power law path loss model is given by

$$
\mathrm{PL}(d)=\mathrm{PL}_{0}+10 n \log _{10}\left(\frac{d}{d_{0}}\right)+X_{\sigma}
$$

where $d$ is the distance between TX and RX, $n$ is the path loss exponent estimated by linear regression, and $X_{\sigma}$ is zeromean Gaussian distributed random variable with standard deviation $\sigma$ and with some time correlation. The $\mathrm{PL}_{0}$ is the path loss at a reference distance $d_{0}$ in $\mathrm{dB}$.

In practice it is observed that a dual-slope model, as stated in [24], can represent measurement data more accurately. We thus characterize a dual-slope model as a piecewiselinear model with the assumption that the power decays with path loss exponent $n_{1}$ and standard deviation $\sigma$ until the breakpoint distance $\left(d_{b}\right)$ and from there it decays with path loss exponent $n_{2}$ and standard deviation $\sigma$. The dual-slope model is given by

$$
\mathrm{PL}(d)= \begin{cases}\mathrm{PL}_{0}+10 n_{1} \log _{10}\left(\frac{d}{d_{0}}\right)+X_{\sigma}, & \text { if } d_{0} \leq d \leq d_{b}, \\ \mathrm{PL}_{0}+10 n_{1} \log _{10}\left(\frac{d_{b}}{d_{0}}\right)+10 n_{2} \log _{10}\left(\frac{d}{d_{b}}\right)+X_{\sigma}, & \text { if } d>d_{b} .\end{cases}
$$

The distance between TX and RX is extracted from the GPS data, which can be unreliable when TX-RX are very close to each other. Moreover, there are only a few samples available for $d<10 \mathrm{~m}$; thus the validity range of the model is set to $d>$ $10 \mathrm{~m}$ and let $d_{0}=10 \mathrm{~m}$. The typical flat earth model considers $d_{b}$ as the distance where the first Fresnel zone touches the ground or where the first signal bounces of the ground that travels $d_{b}+\lambda / 4$ to reach the $\mathrm{RX}$ after reflection. For the measurement setup the height of the TX/RX antennas was $h_{\mathrm{TX}}=h_{\mathrm{RX}}=1.47 \mathrm{~m}$; thus, $d_{b}$ can be calculated as $d_{b}=$ $\left(4 h_{\mathrm{TX}} h_{\mathrm{RX}}-\lambda^{2} / 4\right) / \lambda=161 \mathrm{~m}$ for $\lambda=0.0536 \mathrm{~m}$ at $5.6 \mathrm{GHz}$ carrier frequency. A $d_{b}$ of $104 \mathrm{~m}$ was selected to match the values with the path loss model presented in [24], implying a somewhat better fit to the measurement data.

The path loss exponents before and after $d_{b}$ in (5) are adjusted to fit the median values of the LOS and OLOS data sets in least square sense and are shown in Figures 5 and 6. The extracted parameters are listed in Table 2. For the highway measurements, OLOS occurred only when the TX/RX vehicles were widely separated, that is, when $d>$
$80 \mathrm{~m}$, which means that there are too few samples to model the path loss exponent in OLOS for shorter distances. Thus, the path loss exponent for OLOS for shorter distances is not modeled. Whereas, in practice, this is not always the case, the OLOS can occur at shorter distances if there is traffic congestion on a highway with multiple lanes. The OLOS path loss model presented in this paper has also been verified with the help of an independent empirical analysis [31].

It is interesting to notice that there is an offset $\Delta \mathrm{PL}_{0}$ in the channel gain for the LOS and OLOS data sets, which is of the order of $8.6-10 \mathrm{~dB}$, and is very similar to the results that have previously been reported. In [21] an additional attenuation of $9.6 \mathrm{~dB}$ is attributed to the impact of vehicle as an obstacle. Meireles et al. in [22] stated that the OLOS can cause 10-20 dB of attenuation depending upon traffic conditions, as the congested traffic cause large attenuation. Moreover, it is important to mention that the path loss exponents less than 2 have also been found in many other studies $[9,24,26,32,33]$. It is mainly due to the effect of tworay reflection model in open and highway scenarios or due to wave-guiding in urban scenarios. 


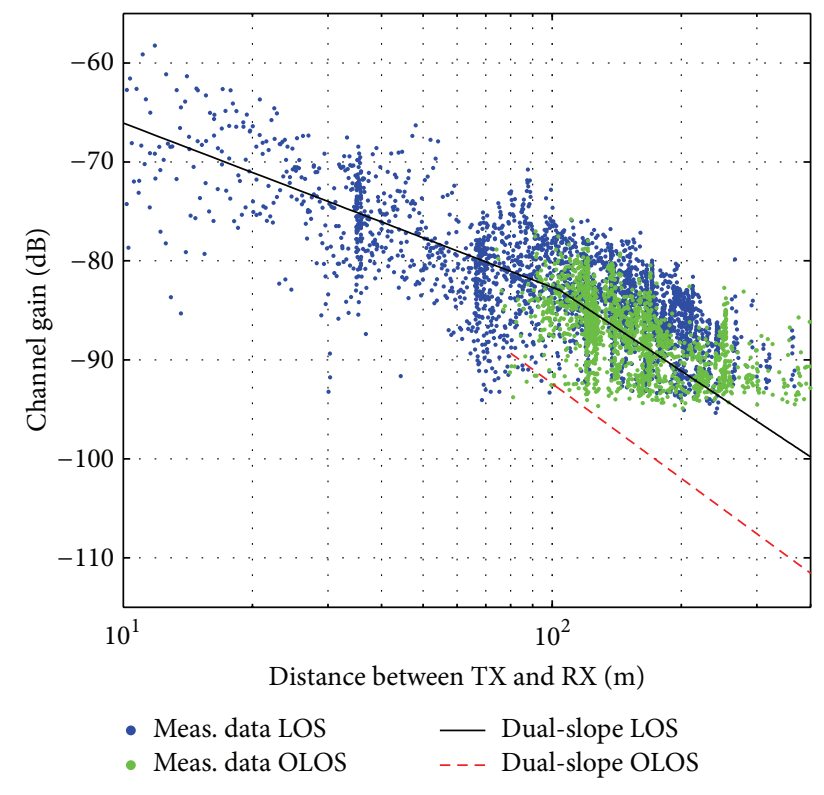

FIGURE 5: Measured channel gain for the highway environment and the least square best fit to the deterministic part of (4).

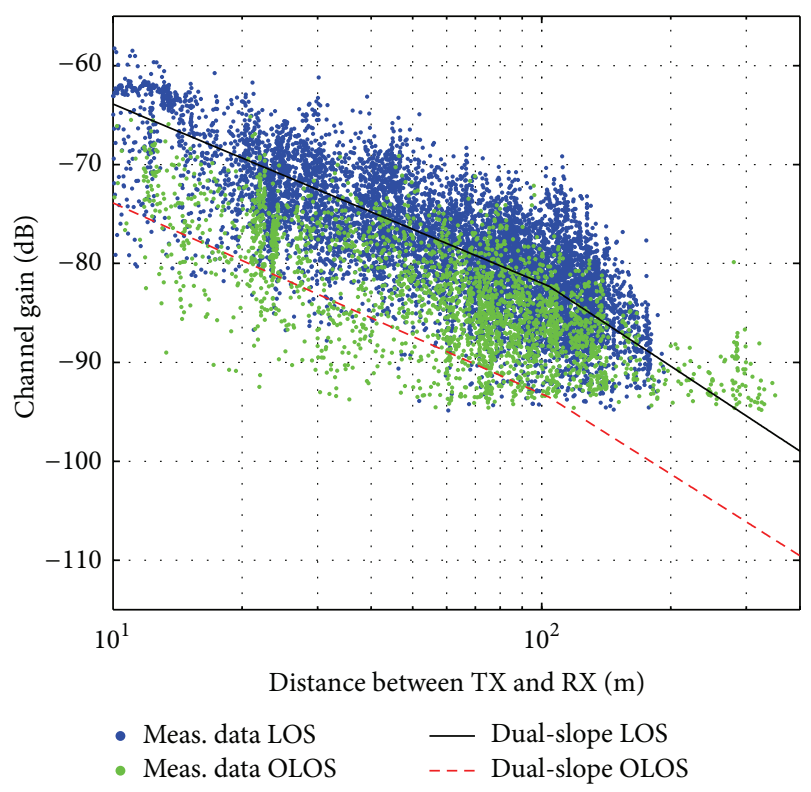

Figure 6: Measured channel gain for the urban environment and the least square best fit to the deterministic part of (4).

TABLE 2: Parameters for the dual-slope path loss model.

\begin{tabular}{lccccc}
\hline & Scenario & $n_{1}$ & $n_{2}$ & $\mathrm{PL}_{0}$ & $\sigma$ \\
\hline \multirow{2}{*}{ LOS } & Highway & -1.66 & -2.88 & -66.1 & 3.95 \\
& Urban & -1.81 & -2.85 & -63.9 & 4.15 \\
\hline \multirow{2}{*}{ OLOS } & Highway & - & -3.18 & -76.1 & 6.12 \\
& Urban & -1.93 & -2.74 & -72.3 & 6.67 \\
\hline
\end{tabular}

It is highly important to model the path loss in the NLOS situation because power level drops quickly when the LOS is blocked by buildings. As mentioned above, the available measured data in NLOS is not sufficient to model the path loss; therefore, it is derived from available models specifically targeting similar scenarios, such as $[12,13,18$, 19, 34] and COST 231-Welfish-Ikegami model (Appendix 7.B in [7]). Among these, Mangel et al. in [13] presented a realistic and a well validated NLOS path loss model which is of low complexity, thus, enabling large-scale packet level simulations in intersection scenarios. The basis for the path loss equation in [13] is a cellular model proposed in [34], which is slightly modified to correspond well to V2V measurements. Validation of the NLOS model using independent $\mathrm{V} 2 \mathrm{~V}$ measurement data is done in [35]; the results show that the model is a good candidate for modeling the path loss in NLOS. For completeness Mangel's model [13] used for the NLOS situation is given as follows:

$$
\begin{aligned}
& \operatorname{PL}\left(d_{r}, d_{t}, w_{r}, x_{t}, i_{s}\right)=3.75+i_{s} 2.94 \\
& + \begin{cases}10 \log _{10}\left(\left(\frac{d_{t}^{0.957}}{\left(x_{t} w_{r}\right)^{0.81}} \frac{4 \pi d_{r}}{\lambda}\right)^{n_{\mathrm{NLOS}}}\right), & \text { if } d_{r} \leq d_{b}, \\
10 \log _{10}\left(\left(\frac{d_{t}^{0.957}}{\left(x_{t} w_{r}\right)^{0.81}} \frac{4 \pi d_{r}^{2}}{\lambda d_{b}}\right)^{n_{\mathrm{NLOS}}}\right), & \text { if } d_{r}>d_{b},\end{cases}
\end{aligned}
$$

where $d_{r} / d_{t}$ are distance of TX/RX to intersection center, $w_{r}$ is width of RX street, $x_{t}$ is distance of TX to the wall, and $i_{s}$ specifies suburban and urban with $i_{s}=1$ and $i_{s}=0$, respectively. In a network simulator, the road topology and TX/RX positions are known; thus, these parameters can be obtained easily. The path loss exponent in NLOS is provided in the model as $n_{\mathrm{NLOS}}=2.69$ and Gaussian distributed fading with $\sigma=4.1 \mathrm{~dB}$.

For larger distances $\left(d_{r}>d_{b}\right)$ the model introduces an increased loss due to diffraction, around the street corners, being dominant. The NLOS model is developed for TX/RX in intersecting streets. If the TX/RX are not in intersecting streets but in parallel streets with buildings blocking the LOS then this NLOS model is not advisable. The direct communication in such setting might not be possible or not required but these vehicles can introduce interference for each other due to diffraction over roof tops. This propagation over the roof top can be approximated by diffraction by multiple screens as it is done in the COST 231 model. However, in [36] simulation results are shown which state that the path loss in parallel street is always very high, $>120 \mathrm{~dB}$. The value is similar to the one obtained with theoretical calculations for diffraction by multiple screens. As the losses for the vehicles in parallel streets are high, interference from such vehicles can simply be ignored.

3.2. Spatial Correlation of Shadow Fading. Once a vehicle goes into a shadow region, it remains shadowed for some time interval implying that the shadowing is a spatially correlated process. If a vehicle is in a shadow region there is possibility that its existence may not be noticed for some time. Hence, it is important to study the spatial correlation of the shadow fading as part of the analysis to find the average decorrelation distances. 
TABLE 3: Decorrelation distances $d_{c}$ for highway and urban scenarios.

\begin{tabular}{lcc}
\hline Scenario & LOS & OLOS \\
\hline Highway & 23.3 & 32.5 \\
Urban & 4.25 & 4.5 \\
\hline
\end{tabular}

The large-scale variation of shadow fading can be well described as a Gaussian random variable (discussed in Section 3). By subtracting the distance dependent mean from the overall channel gain, the shadow fading can be assumed to be stationary process. Then the spatial autocorrelation of the shadow fading can be written as

$$
r_{x}(\Delta d)=E\left\{X_{\sigma} X_{\sigma}(d+\Delta d)\right\} .
$$

The autocorrelation of the Gaussian process can then be modeled by a well-known analytical model proposed by Gudmundson [37], which is a simple negative exponential function,

$$
r_{x}(\Delta d)=e^{-|\Delta d| / d_{c}},
$$

where $\Delta d$ is an equally spaced distance vector and $d_{c}$ is a decorrelation distance being a scenario-dependent real valued constant. In the Gudmundson model, $d_{c}$ is defined as the value of $\Delta d$ at which the value of the autocorrelation function $r_{x}(\Delta d)$ is equal to $1 / e$. The value of the decorrelation distance $d_{c}$ is determined from both the LOS and OLOS measured autocorrelation functions and are given in Table 3, for both highway and urban scenarios, respectively. The estimated correlation distance is thus used to model the measured autocorrelation functions using (8) and is shown in Figures 7(a) and 7(b).

Looking at decorrelation distance $d_{c}$, the implementation of shadow fading in a simulator can, if desired, be simplified by treating it as a block shadow fading, where $d_{c}$ can be assumed as a block length in which the signal power will remain, more or less, constant.

3.3. Extension in the Traffic Mobility Models. The mobility models of today implemented in VANET simulators are very advanced; SUMO (Simulation of Urban Motility) [38] is one example of such an open source mobility model. These advanced models are capable of taking into account vehicle positions, exact speeds, intervehicle spacings, accelerations, overtaking attitudes, lane-change behaviors, and so forth. However, the ability to treat the vehicles as obstacles and model the intensity at which they obstruct the LOS for other vehicles is currently missing. Therefore, an extension for including shadowing effects in network simulators is provided herein, as stated in [39]. Since the vehicular mobility models implemented in the simulators give instantaneous information about each vehicle, the state of TX and RX vehicles can be identified by a simple geometric manipulation in the existing traffic mobility models as follows.

(i) Model each vehicle or building as a rectangle in the simulator.

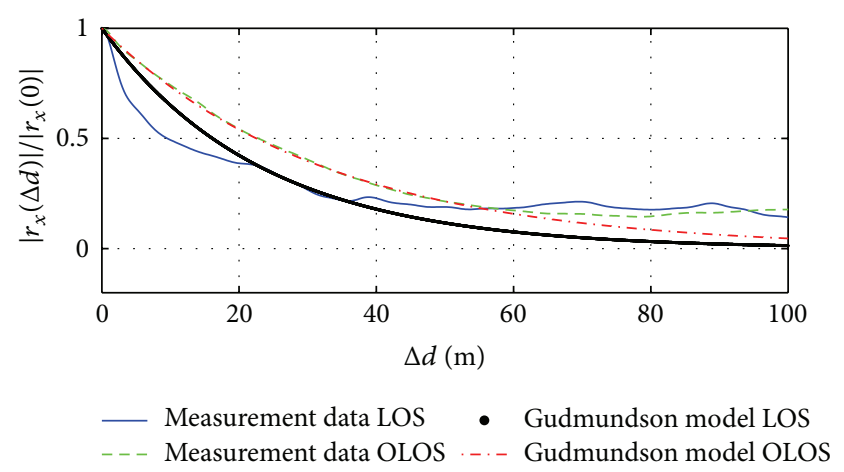

(a)

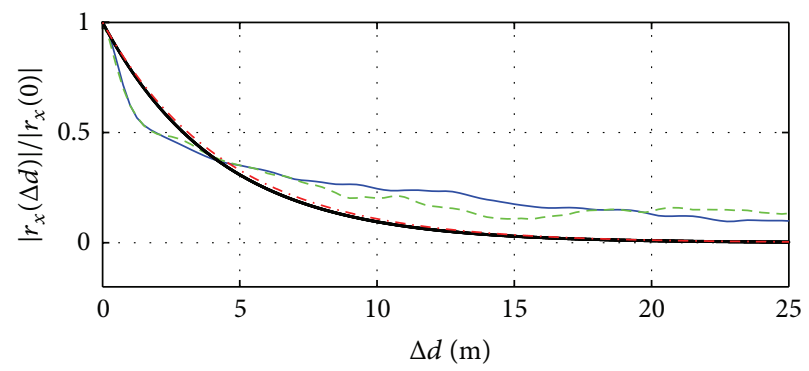

— Measurement data LOS - Gudmundson model LOS Measurement data OLOS Gudmundson model OLOS

(b)

FIGURE 7: Measured autocorrelation function and model according to (8) for LOS and OLOS data: (a) highway scenario and (b) urban scenarios.

(ii) Draw a straight line starting from the antenna position of each TX vehicle to the antenna position of each RX vehicle.

(iii) If the line does not touch any other rectangle, TX/RX has LOS.

(iv) If the line passes through another rectangle, the LOS is obstructed by a vehicle or by a building, the two cases are distinguished by using the geographical information available in the simulator.

(v) Once the propagation condition is identified, the simulator can simply use the relevant model to calculate the power loss.

The impact of an obstacle is usually assessed qualitatively by the concept of the Fresnel ellipsoids. Only the visual sight does unfortunately not promise the availability of LOS; it is required that the Fresnel zone is free of obstacles in order to have the LOS [7]. The availability of LOS based on Fresnel ellipsoids depends very much on the information about the height of the obstacle, its distance from TX and RX, the distance between TX and RX, and the wavelength $\lambda$. The information, if available in the traffic mobility simulator, should be utilized for the characterization of LOS and OLOS situation. 


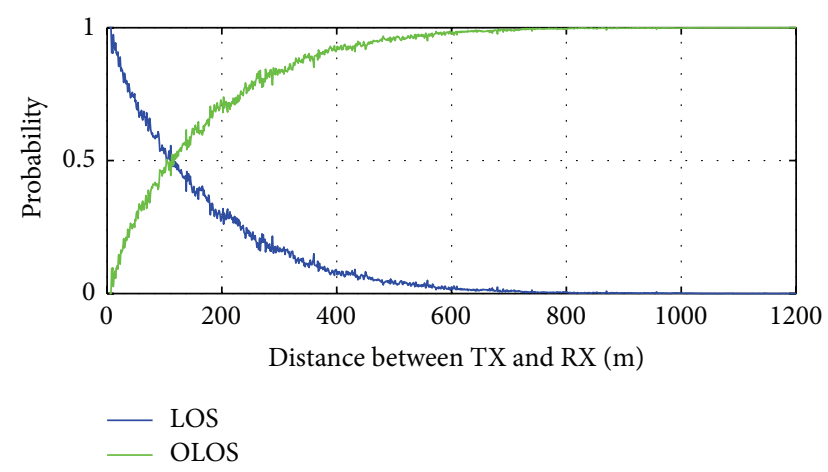

FIGURE 8: The probabilities of being in LOS and OLOS, respectively, depending on distance between TX and RX.

\section{Network Simulations}

Finally, networks simulations are provided to show the difference between Cheng's Nakagami model [24] and the channel model presented herein distinguishing between LOS and OLOS. The simulation scenario is a $10 \mathrm{~km}$ long highway with four lanes (two in each direction). The vehicles appear with a Poisson distribution with three different simulation settings for the mean interarrival time: $1 \mathrm{~s}, 2 \mathrm{~s}$, and $3 \mathrm{~s}$. The three different mean interarrival times yield three different vehicle densities, where $1 \mathrm{~s}$ corresponds to $\approx 100$ vehicles $/ \mathrm{km}$, $2 \mathrm{~s}$ corresponds to 60 vehicles/ $\mathrm{km}$, and $3 \mathrm{~s}$ corresponds to 40 vehicles/km. Every vehicle broadcasts 400 -byte long position messages at $10 \mathrm{~Hz}(10$ messages/sec) using a transfer rate of $6 \mathrm{Mbps}$ and an output power of $20 \mathrm{dBm}(100 \mathrm{~mW})$. The channel access procedure is carrier sense multiple access (CSMA) that has been selected as medium access control (MAC) for VANETs supporting road traffic safety applications. The vehicle speeds are independently Gaussian distributed with a standard deviation of $1 \mathrm{~m} / \mathrm{s}$, with different means $(23 \mathrm{~m} / \mathrm{s}$ and $30 \mathrm{~m} / \mathrm{s}$ ) depending on lane. The vehicles maintain the same speed as long as they are on the highway. More details about the simulator can be found in [40]. The shadowing based channel LOS/OLOS model presented herein has been compared against Cheng's Nakagami model [24] in the network simulations, where the latter is not capable of distinguishing between LOS and OLOS. Cheng's Nakagami model is also based on an outdoor channel sounding campaign, performed at $5.9 \mathrm{GHz}$ in which the small-scale fading and the shadowing are both represented by the Nakagami- $m$ model [24]. The fading intensities, represented by the $m$ parameter of the Nakagami distribution, are different depending on the distance between TX and RX. The $m$ values and the path loss exponents are taken from data set 1 in [24] to compute the averaged received power for Cheng's Nakagami model.

The averaged received power for the LOS/OLS and Cheng's Nakagami model is depicted in Figure 9. At shorter distances there is a little chance that another vehicle is between two communicating vehicles but as the distance increases the chances of being under OLOS by either vehicle or object or due to the curvature of the earth increase. The probabilities of being in LOS, Prob(LOS $\mid d$ ), and being

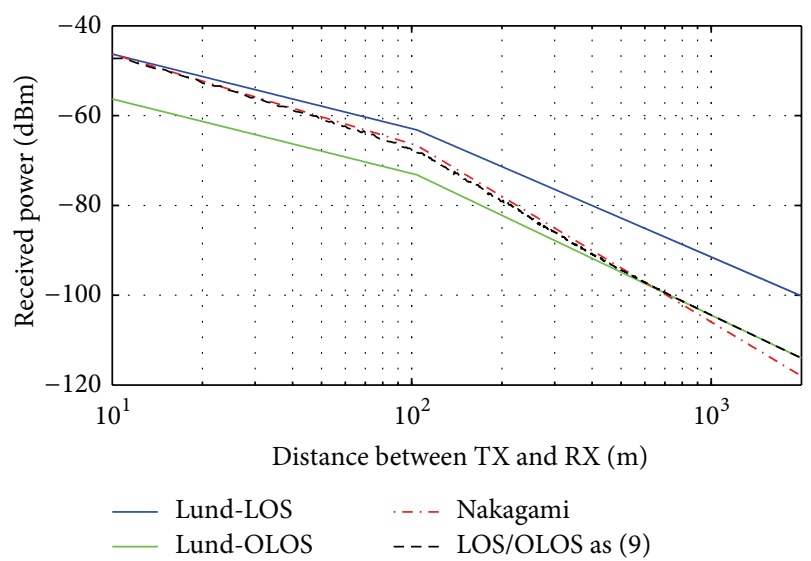

FIGURE 9: The averaged received power for the LOS/OLOS model and Cheng's Nakagami model, respectively.

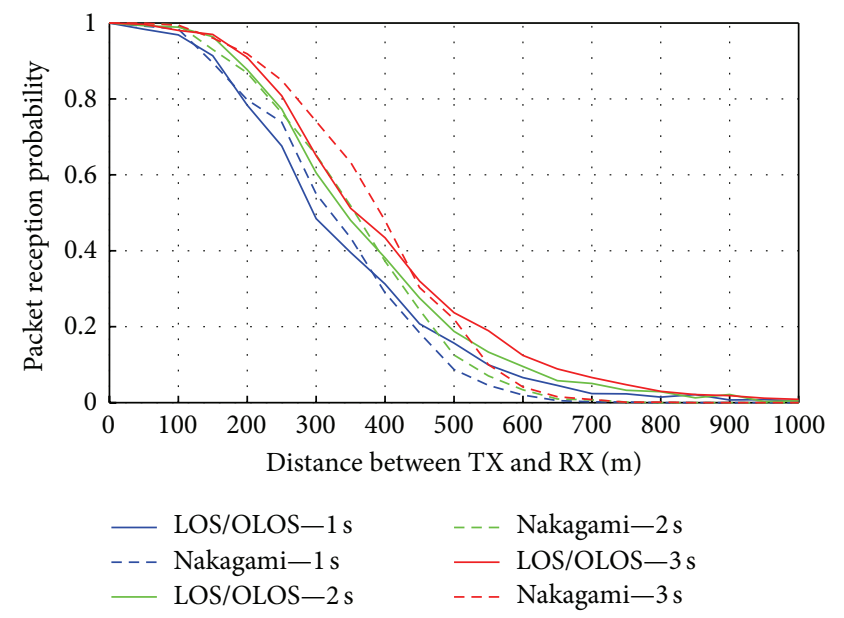

FIGURE 10: Packet reception probability for the two different channel models and for three different vehicle densities.

in OLOS, Prob(OLOS $\mid d$ ), have been calculated from the network simulator for the highway scenario, as a function of distance and are depicted in Figure 8. To receive the averaged power as a function of distance similar to Cheng's model, these probabilities can be multiplied with the averaged received power for LOS, $P_{\mathrm{RX}, \mathrm{LOS}}(d)$, and OLOS, $P_{\mathrm{RX}, \mathrm{OLOS}}(d)$, using the following equation:

$$
\begin{aligned}
P_{\mathrm{RX}}(d)= & \operatorname{Prob}(\mathrm{LOS} \mid d) P_{\mathrm{RX}, \mathrm{LOS}}(d) \\
& +\operatorname{Prob}(\mathrm{OLOS} \mid d) P_{\mathrm{RX}, \mathrm{OLOS}}(d) .
\end{aligned}
$$

By using (9) the averaged received power from the LOS/OLOS model coincides with Cheng's Nakagami model (see Figure 9), which is very interesting to notice. In Figure 10, the packet reception probability is depicted for the two channel models, LOS/OLOS model and Cheng's Nakagami model, respectively, and for three different vehicle densities. On the $x$-axis, the distance between TX and RX is shown. For detailed analysis six pairs of vehicles, three pairs in each direction are studied in the simulations. Each pair 


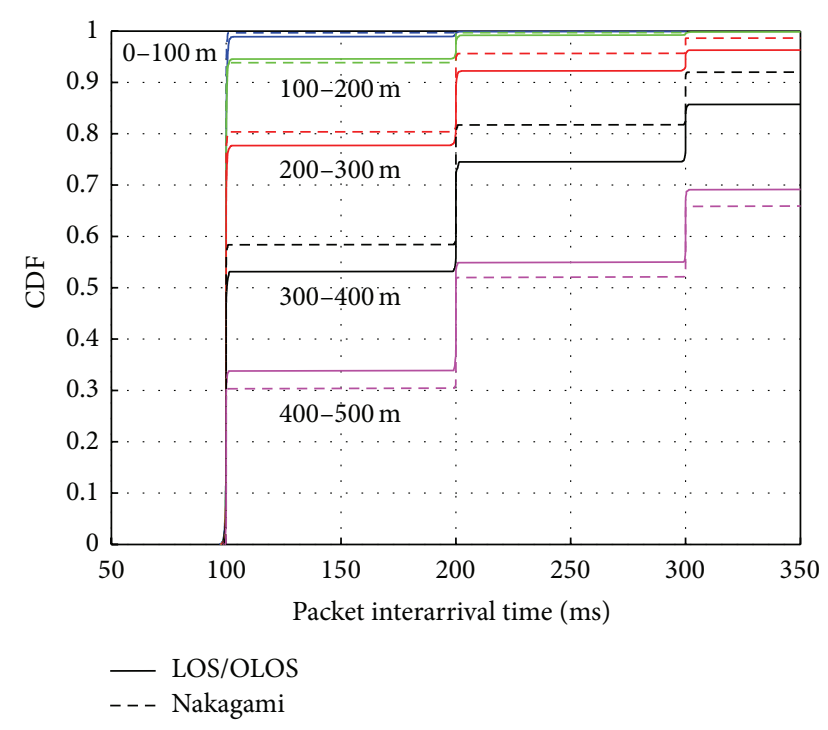

FIgURE 11: CDF for the packet interarrival time for a vehicle density of 40 vehicles/ $\mathrm{km}$ for the LOS/OLOS and Nakagami models, respectively.

travels in the same direction in different lanes with different speeds, where the vehicle with high speed will pass by the vehicle with the low speed. The selection of pairs was done to study individual performance of the vehicles. It should be noted that exactly the same number of communicating vehicles has been used for the different vehicle densities for every channel model; that is, the same TX-RX pair are studied using both channel models. When TX and RX are close to each other, that is, within $100 \mathrm{~m}$, the two channel models perform equally. As the distance increases to $200-400 \mathrm{~m}$, the vehicles exposed to the Nakagami model are experiencing a better packet reception probability. The vehicles exposed to the Nakagami model reach a packet reception probability of zero at around $700 \mathrm{~m}$, whereas this reached above $1000 \mathrm{~m}$ for the LOS/OLOS model. Real measurements [41] also show such behavior with occasional successful packet transmission at larger distances. This also implies that the LOS/OLOS model contributes to interference at stations situated further away, which is in line with what is seen on the averaged received power in Figure 9. Here the OLOS part has a stronger signal than Cheng's Nakagami model above $700 \mathrm{~m}$.

In Figure 11, the CDF for packet interarrival time for a vehicle density of 3 seconds ( 40 vehicles $/ \mathrm{km}$ ) is depicted. The received packet interarrival time is the time that has elapsed between two successfully received packets from a specific TX that RX is listening to. Around every $100 \mathrm{~ms}$, the RX is expecting a new packet from a specific TX. The period is not exactly $100 \mathrm{~ms}$ due to channel access delays caused by, for example, backoff procedures [42]. The different lines in the figure represent different distance bins. When TX and RX are within $100 \mathrm{~m}$ of each other, the RX can expect a new packet every $100 \mathrm{~ms}$ and the channel models only differ slightly in performance. When distance increases to $200-400 \mathrm{~m}$, the vehicles under the treatment of the Nakagami model are

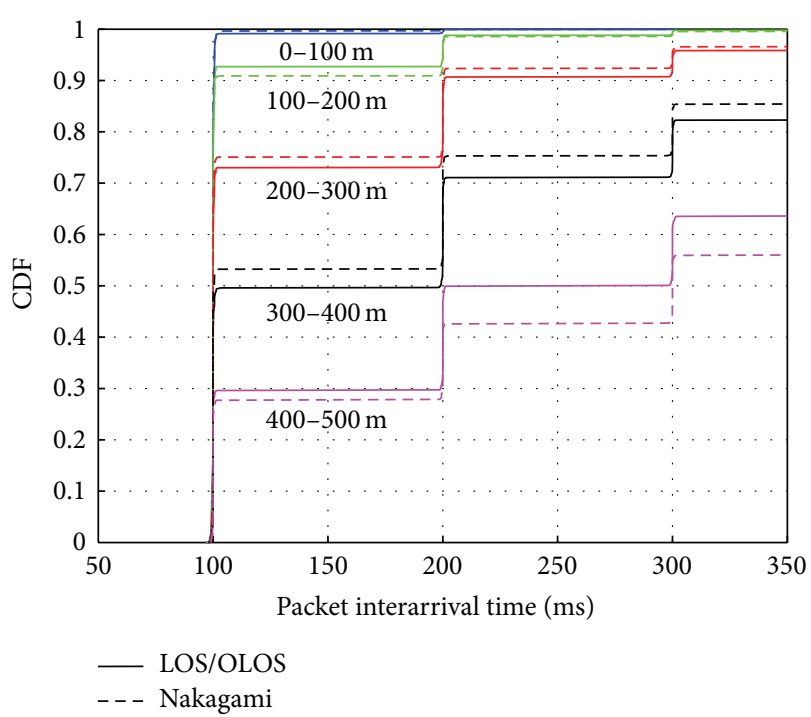

FIGURE 12: CDF for the packet interarrival time for a vehicle density of 60 vehicles/km for the LOS/OLOS and Nakagami models, respectively.

experiencing a better packet interarrival times, which was also reflected in Figure 10. In the distance bin 300-400 m, studying the vehicles under Nakagami, in almost $60 \%$ of the cases there are no packets lost between two successful receptions and, in about $25 \%$ of the cases, a single packet is lost between successful receptions. As the distance increases to above $400 \mathrm{~m}$, stations under the LOS/OLOS model are having slightly better packet reception probabilities. The CDF for packet interarrival times for a vehicle density of $2 \mathrm{~s}$ (60 vehicles $/ \mathrm{km}$ ) is depicted in Figure 12. Here, it is seen that the gap in performance between the two models has decreased since there are more transmissions on the channel and the overall interference has increased. In Figure 13, $\mathrm{CDF}$ for packet interarrival times for the highest vehicle density case is depicted. Still, the stations under the treatment of Nakagami are experiencing a better packet interarrival pattern, especially for distances between 200 and $300 \mathrm{~m}$.

\section{Summary and Conclusions}

In this paper, a shadow fading model based on measurements performed in urban and highway scenarios is presented, where a separation between LOS, obstructed LOS by vehicle (OLOS), and obstructed LOS by building (NLOS), is performed. In the past, despite extensive research efforts to develop more realistic channel models for $\mathrm{V} 2 \mathrm{~V}$ communication, the impact of vehicles obstructing LOS has largely been ignored. We have observed that the LOS obstruction by vehicles (OLOS) induce an additional loss, of about $10 \mathrm{~dB}$, in the received power. Network simulations have been conducted showing the difference between a conventional Nakagami based channel model (often used in VANET simulations) and the LOS/OLOS model presented herein. There is a difference in the performance of the two channel models. However, depending on the evaluated VANET application 


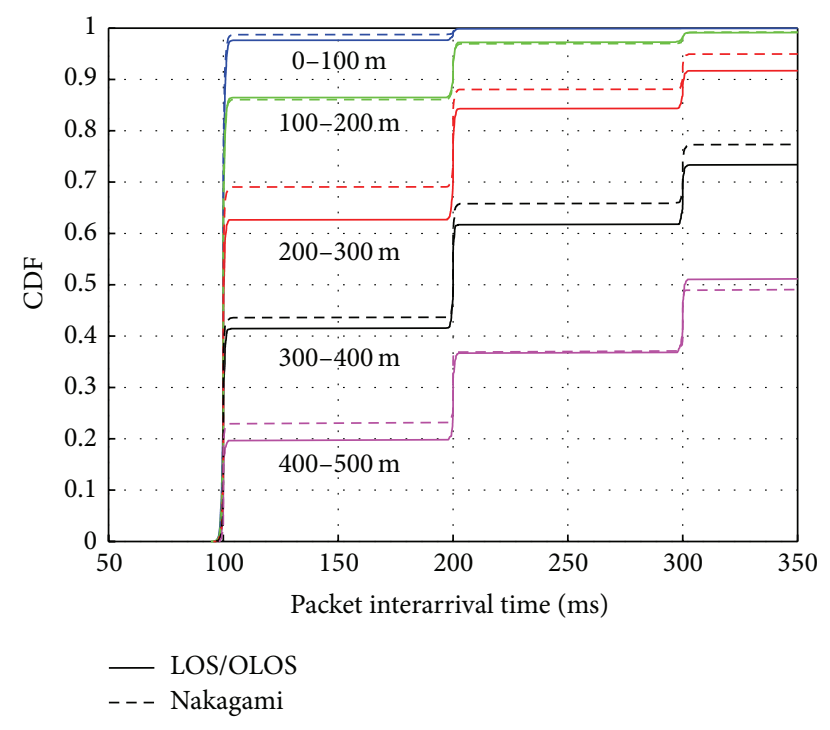

FIGURE 13: CDF for the packet interarrival time for a vehicle density of 100 vehicles/km for the LOS/OLOS and Nakagami models, respectively.

the obstruction of LOS cannot be ignored and there is a need for a LOS/OLOS model in VANET simulators. The LOS/OLOS model is easy to implement in VANET simulators due to the usage of a dual-piece wise path loss model and the shadowing effect is modeled as a log-normal correlated variable with a mean determined by the propagation condition (LOS/OLOS).

\section{Conflict of Interests}

The authors declare that there is no conflict of interests regarding the publication of this paper.

\section{Acknowledgments}

This work was partially funded by the Excellence Center at Linköping-Lund in Information Technology (ELLIIT) and partially funded by Higher Education Commission (HEC) of Pakistan.

\section{References}

[1] J. Gozalvez, M. Sepulcre, and R. Bauza, "Impact of the radio channel modelling on the performance of VANET communication protocols," Telecommunication Systems, vol. 50, no. 3, pp. 149-167, 2012.

[2] G. Acosta-Marum and M. A. Ingram, "Six time- and frequencyselective empirical channel models for vehicular wireless LANs," IEEE Vehicular Technology Magazine, vol. 2, no. 4, pp. 4-11, 2007.

[3] I. Sen and D. W. Matolak, "Vehicle-vehicle channel models for the $5 \mathrm{GHz}$ band," IEEE Transactions on Intelligent Transportation Systems, vol. 9, no. 2, pp. 235-245, 2008.

[4] A. Paier, J. Karedal, N. Czink et al., "Characterization of vehicleto-vehicle radio channels from measurements at $5.2 \mathrm{GHz}$,"
Wireless Personal Communications, vol. 50, no. 1, pp. 19-32, 2009.

[5] J. S. Otto, F. E. Bustamante, and R. A. Berry, "Down the block and around the corner: the impact of radio propagation on inter-vehicle wireless communication," in Proceedings of the 29th IEEE International Conference on Distributed Computing Systems Workshops (ICDCS '09), pp. 605-614, June 2009.

[6] O. Renaudin, V.-M. Kolmonen, P. Vainikainen, and C. Oestges, "Non-stationary narrowband MIMO inter-vehicle channel characterization in the $5-\mathrm{GHz}$ band," IEEE Transactions on Vehicular Technology, vol. 59, no. 4, pp. 2007-2015, 2010.

[7] A. Molisch, Wireless Communications, IEEE Press-Wiley, Chichester, UK, 2005.

[8] T. Abbas, J. Karedal, F. Tufvesson, A. Paier, L. Bernadó, and A. F. Molisch, "Directional analysis of vehicle-to-vehicle propagation channels," in Proceedings of the IEEE 73rd Vehicular Technology Conference (VTC-Spring '11), pp. 1-5, May 2011.

[9] A. F. Molisch, F. Tufvesson, J. Karedal, and C. F. Mecklenbräuker, "A survey on vehicle-to-vehicle propagation channels," IEEE Wireless Communications, vol. 16, no. 6, pp. 12-22, 2009.

[10] T. Abbas, L. Bernado, A. Thiel, C. Mecklenbrauker, and F. Tufvesson, "Radio channel properties for vehicular communication: merging lanes versus urban intersections," IEEE Vehicular Technology Magazine, vol. 8, no. 4, pp. 27-34, 2013.

[11] T. Mangel, M. Michl, O. Klemp, and H. Hartenstein, "Realworld measurements of non-line-of-sight reception quality for $5.9 \mathrm{GHz}$ IEEE 802.11p at intersections," in Communication Technologies for Vehicles, vol. 6596 of Lecture Notes in Computer Science, pp. 189-202, Springer, Berlin, Germany, 2011.

[12] E. Giordano, R. Frank, G. Pau, and M. Gerla, "CORNER: a realistic urban propagation model for VANET," in Proceedings of the 7th International Conference on Wireless On-demand Network Systems and Services (WONS'10), pp. 57-60, Kranjska Gora, Slovenia, February 2010.

[13] T. Mangel, O. Klemp, and H. Hartenstein, " $5.9 \mathrm{GHz}$ intervehicle communication at intersections: a validated non-lineof-sight path-loss and fading model," EURASIP Journal on Wireless Communications and Networking, vol. 2011, no. 1, article 182, 2011.

[14] P. Paschalidis, K. Mahler, A. Kortke, M. Peter, and W. Keusgen, "Pathloss and multipath power decay of the wideband carto-car channel at $5.7 \mathrm{GHz}$," in Proceedings of the IEEE $73 \mathrm{rd}$ Vehicular Technology Conference (VTC '11), Budapest, Hungry, May 2011.

[15] J. Turkka and M. Renfors, "Path loss measurements for a nonline-of-sight mobile-to-mobile environment," in Proceedings of the 8th International Conference on Intelligent Transport System Telecommunications (ITST '08), pp. 274-278, Hilton Phuket, Thailand, October 2008.

[16] K. Mahler, P. Paschalidis, M. Wisotzki, A. Kortke, and W. Keusgen, "Evaluation of vehicular communication performance at street intersections," in Proceedings of the IEEE 80th Vehicular Technology Conference (VTC'14), pp. 1-5, Vancouver, Canada, September 2014.

[17] Z. Xu, L. Bernado, M. Gan et al., "Relaying for IEEE 802.11p at road intersection using a vehicular non-stationary channel model," in Proceedings of the IEEE 6th International Symposium on Wireless Vehicular Communications (WiVeC' 14), pp. 1-6, Vancouver, Canada, September 2014.

[18] O. Onubogu, K. Ziri-Castro, D. Jayalath, K. Ansari, and H. Suzuki, "Empirical vehicle-to-vehicle pathloss modeling in 
highway, suburban and urban environments at $5.8 \mathrm{GHz}$," in Proceedings of the 8th International Conference on Signal Processing and Communication Systems (ICSPCS '14), pp. 1-6, Gold Coast, Australia, December 2014.

[19] L. Urquiza-Aguiar, C. Tripp-Barba, J. Estrada-Jiménez, and M. A. Igartua, "On the impact of building attenuation models in vanet simulations of urban scenarios," Electronics, vol. 4, no. 1, pp. 37-58, 2015.

[20] Y. Zang, L. Stibor, G. Orfanos, S. Guo, and H.-J. Reumerman, "An error model for inter-vehicle communications in highway scenarios at $5.9 \mathrm{GHz}$," in Proceedings of the 2nd ACM International Workshop on Performance Evaluation of Wireless Ad Hoc, Sensor, and Ubiquitous Networks (PE-WASUN' 05), pp. 49-56, ACM, New York, NY, USA, 2005.

[21] M. Boban, T. T. V. Vinhoza, M. Ferreira, J. Barros, and O. K. Tonguz, "Impact of vehicles as obstacles in Vehicular Ad Hoc Networks," IEEE Journal on Selected Areas in Communications, vol. 29, no. 1, pp. 15-28, 2011.

[22] R. Meireles, M. Boban, P. Steenkiste, O. Tonguz, and J. Barros, "Experimental study on the impact of vehicular obstructions in VANETs," in Proceedings of the IEEE Vehicular Networking Conference (VNC '10), pp. 338-345, IEEE, Jersey City, NJ, USA, December 2010.

[23] M. Boban, J. Barros, and O. K. Tonguz, "Geometry-based vehicle-to-vehicle channel modeling for large-scale simulation," IEEE Transactions on Vehicular Technology, vol. 63, no. 9, pp. 4146-4164, 2014.

[24] L. Cheng, B. E. Henty, D. D. Stancil, F. Bai, and P. Mudalige, "Mobile vehicle-to-vehicle narrow-band channel measurement and characterization of the $5.9 \mathrm{GHz}$ Dedicated Short Range Communication (DSRC) frequency band," IEEE Journal on Selected Areas in Communications, vol. 25, no. 8, pp. 1501-1516, 2007.

[25] T. Abbas, Measurement based channel characterization and modeling for vehicle-to-vehicle communications [Ph.D. thesis], Lund University, 2014.

[26] J. Karedal, N. Czink, A. Paier, F. Tufvesson, and A. F. Molisch, "Path loss modeling for vehicle-to-vehicle communications," IEEE Transactions on Vehicular Technology, vol. 60, no. 1, pp. 323-328, 2011.

[27] L. Bernadó, T. Zemen, F. Tufvesson, A. F. Molisch, and C. F. Mecklenbräuker, "The (in-) validity of the WSSUS assumption in vehicular radio channels," in Proceedings of the IEEE 23rd International Symposium on Personal, Indoor and Mobile Radio Communications (PIMRC '12), pp. 1757-1762, September 2012.

[28] J. Karedal, F. Tufvesson, N. Czink et al., "A geometry-based stochastic MIMO model for vehicle-to-vehicle communications," IEEE Transactions on Wireless Communications, vol. 8, no. 7, pp. 3646-3657, 2009.

[29] G. Stüber, Principles of Mobile Communication, Kluwer Academic, Dordrecht, The Netherlands, 2nd edition, 2000.

[30] A. P. Dempster, N. M. Laird, and D. B. Rubin, "Maximum likelihood from incomplete data via the EM algorithm," Journal of the Royal Statistical Society, Series B: Methodological, vol. 39, no. 1, pp. 1-38, 1977.

[31] D. Vlastaras, T. Abbas, M. Nilsson, R. Whiton, M. Olback, and F. Tufvesson, "Impact of a truck as an obstacle on vehicle-tovehicle communications in rural and highway scenarios," in Proceedings of the IEEE 6th International Symposium on Wireless Vehicular Communications (WiVeC '14), pp. 1-6, Vancouver, Canada, September 2014.
[32] H. Fernández, L. Rubio, J. Reig, V. Rodrigo-Peñarrocha, and A. Valero, "Path loss modeling for vehicular system performance and communication protocols evaluation," Mobile Networks and Applications, vol. 18, no. 6, pp. 755-765, 2013.

[33] J. Kunisch and J. Pamp, "Wideband car-to-car radio channel measurements and model at $5.9 \mathrm{GHz}$," in Proceedings of the IEEE 68th Vehicular Technology Conference (VTC' 08), pp. 1-5, Calgary, Canada, September 2008.

[34] H. El-Sallabi, "Fast path loss prediction by using virtual source technique for urban microcells," in Proceedings of the IEEE 51st Vehicular Technology Conference (VTC '00), vol. 3, pp. 21832187, Tokyo, Japan, 2000.

[35] T. Abbas, A. Thiel, T. Zemen, C. F. Mecklenbräuker, and F. Tufvesson, "Validation of a non-line-of-sight path-loss model for V2V communications at street intersections," in Proceedings of the 13th International Conference on ITS Telecommunications (ITST '13), pp. 198-203, IEEE, Tampere, Finland, November 2013.

[36] T. Gaugel, L. Reichardt, J. Mittag, T. Zwick, and H. Hartenstein, "Accurate simulation of wireless vehicular networks based on ray tracing and physical layer simulation," in High Performance Computing in Science and Engineering '11, pp. 619-630, Springer, Berlin, Germany, 2012.

[37] M. Gudmundson, "Correlation model for shadow fading in mobile radio systems," Electronics Letters, vol. 27, no. 23, pp. 2145-2146, 1991.

[38] M. Behrisch, L. Bieker, J. Erdmann, and D. Krajzewicz, "SUMO-simulation of urban mobility: an overview," in Proceedings of the 3rd International Conference on Advances in System Simulation (SIMUL' 11), pp. 63-68, Barcelona, Spain, October 2011.

[39] T. Abbas and F. Tufvesson, "Line-of-sight obstruction analysis for vehicle-to-vehicle network simulations in a two-lane highway scenario," International Journal of Antennas and Propagation, vol. 2013, Article ID 459323, 9 pages, 2013.

[40] K. Sjöberg, Medium access control in vehicular ad hoc networks [Ph.D. thesis], Chalmers University of Technology, Goteborg, Sweden, 2013.

[41] A. Paier, The vehicular radio channel in the $5 \mathrm{GHz}$ band [Ph.D. thesis], Technische Universität Wien, Vienna, Austria, 2010.

[42] IEEE Std. 802.11p-2010, Part 11: Wireless LAN Medium Access Control (MAC) and Physical Layer (PHY) Specifications: Amendment 7: Wireless Access in Vehicular Environment, IEEE Std., July 2010. 

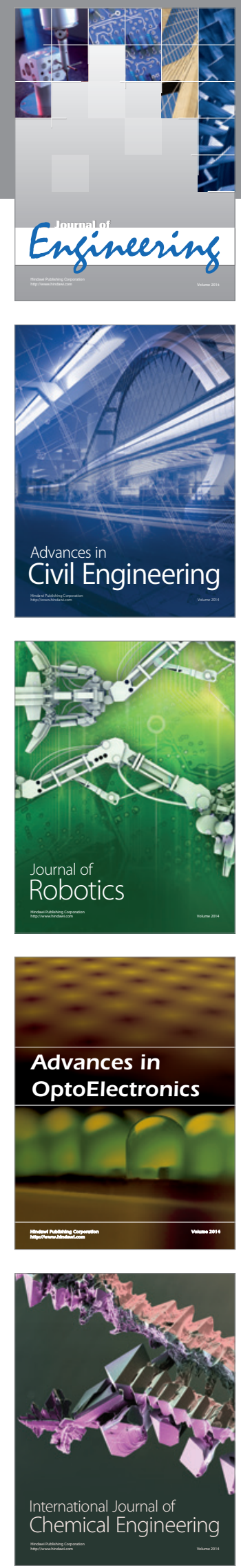

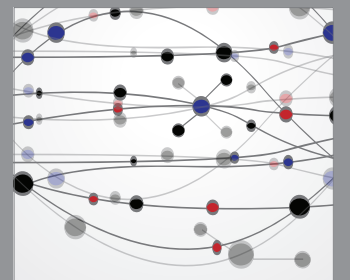

The Scientific World Journal
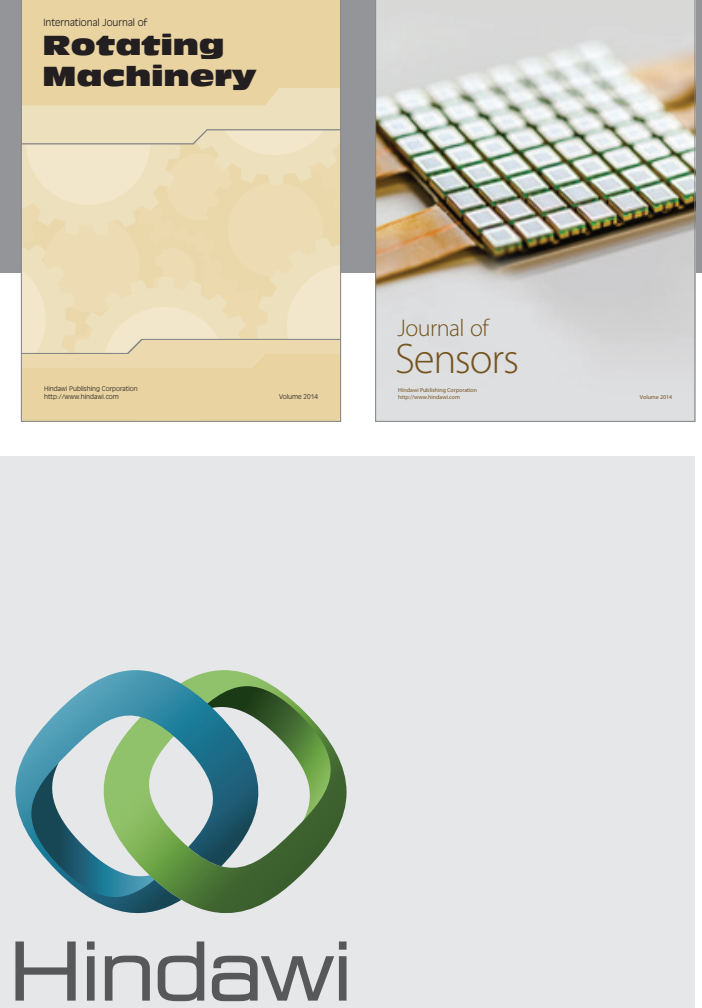

Submit your manuscripts at http://www.hindawi.com
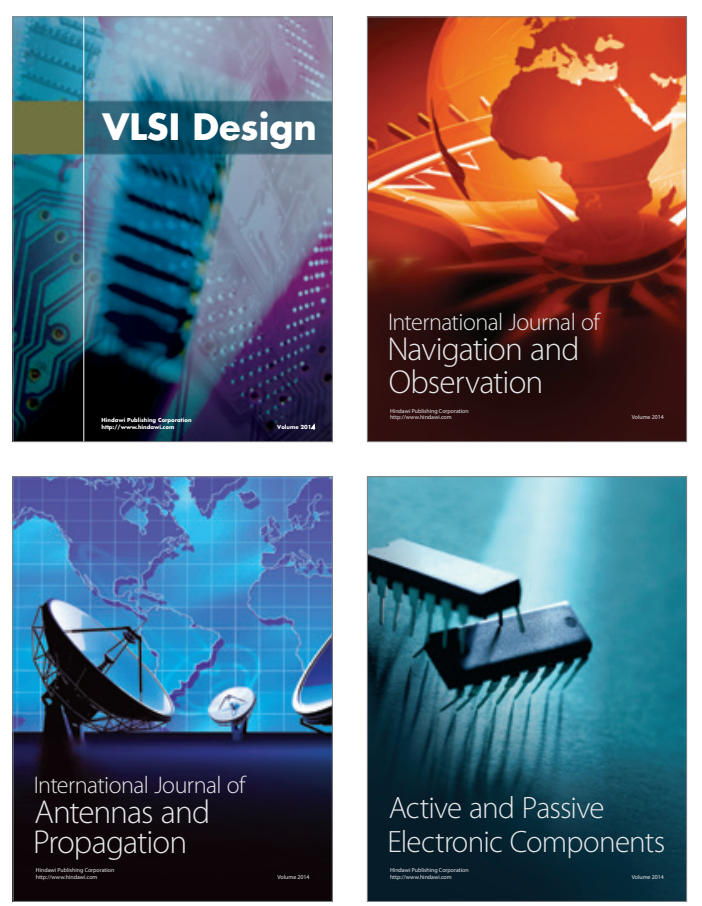
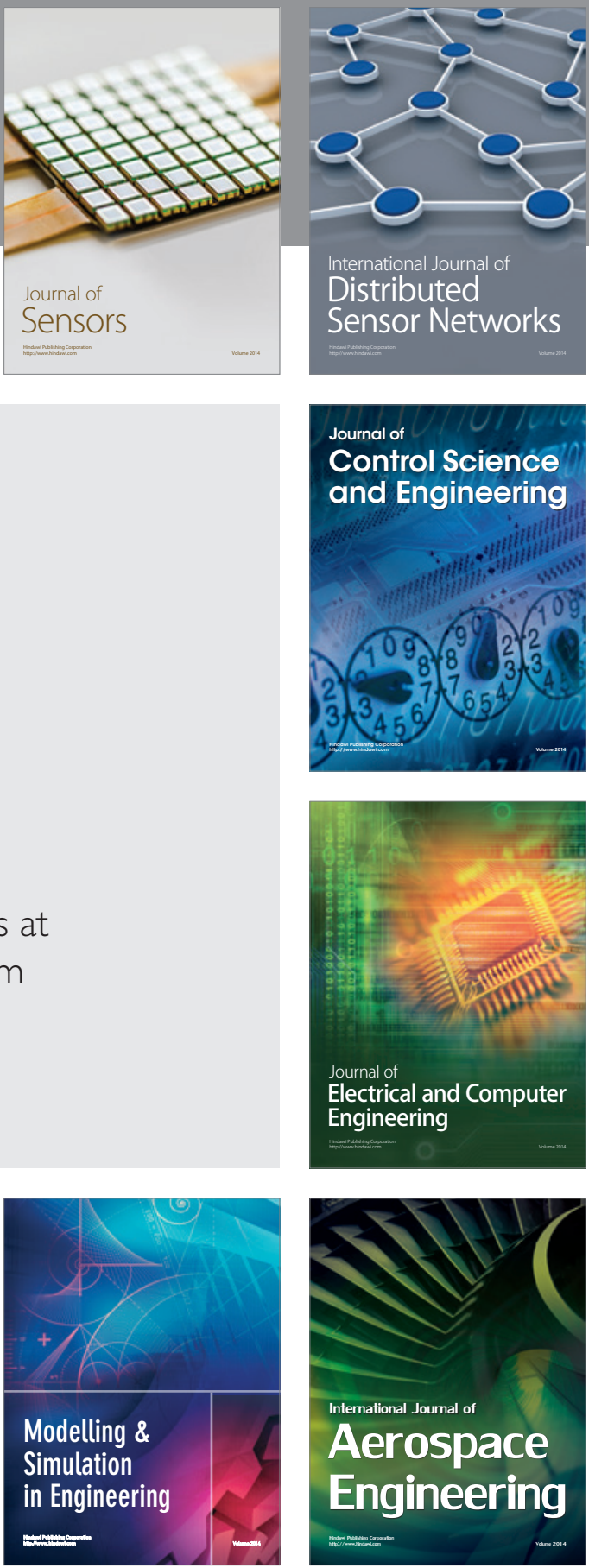

Journal of

Control Science

and Engineering
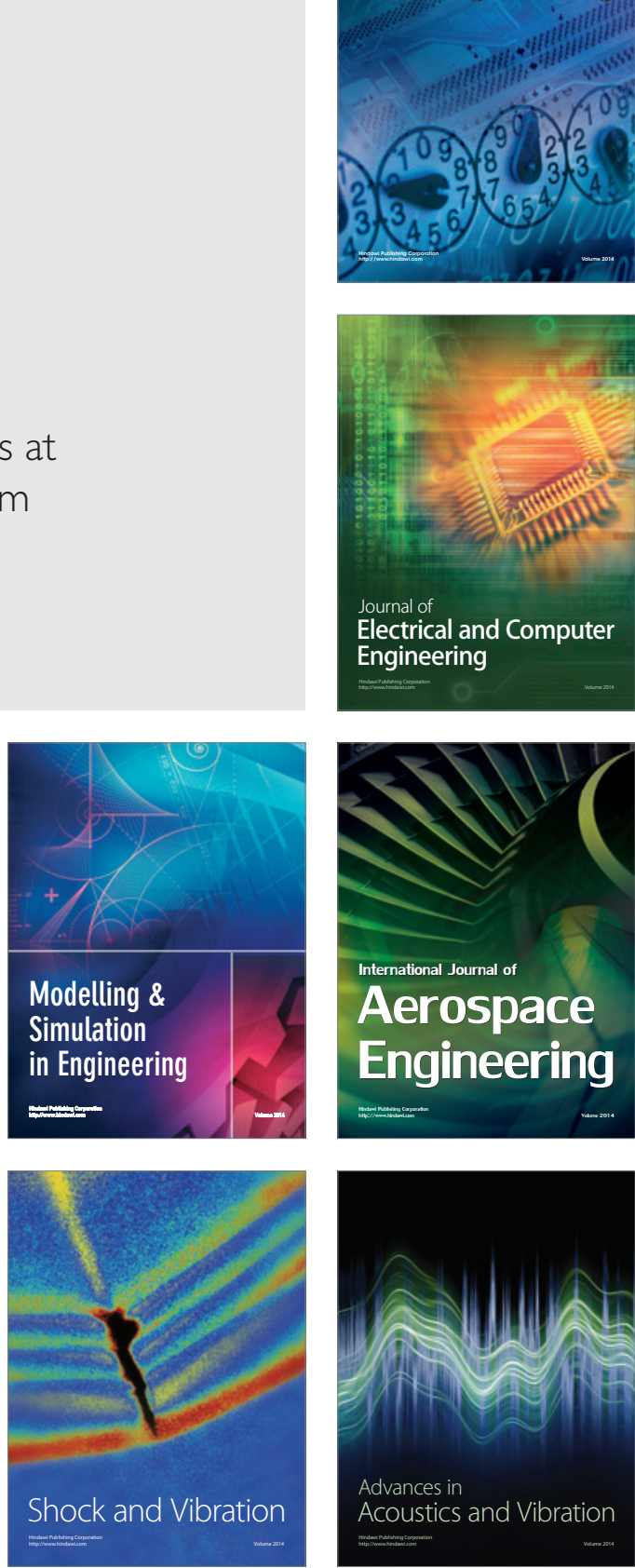